Electron Cloud Observations at RHIC in Run-3 (2002/03)

U. Iriso, A.K. Drees, W. Fischer, D. Gassner, O. Gould, J. Gullotta, P. He, H.C. Hseuh, R. Lee, V. Ponnaiyan, L. Smart, D. Trbojevic and S.Y. Zhang

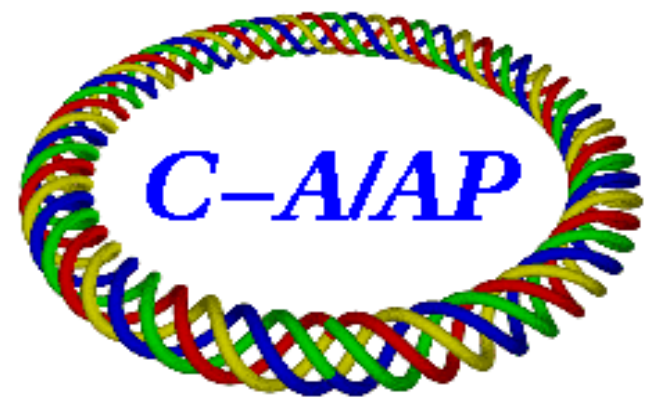

Collider-Accelerator Department Brookhaven National Laboratory Upton, NY 11973 


\title{
ELECTRON CLOUD OBSERVATIONS AT RHIC IN RUN-3 (2002/03)
}

\author{
U. Iriso, A.K. Drees, W. Fischer, D. Gassner, O. Gould, \\ J. Gullotta, P. He, H.C. Hseuh, R. Lee, V. Ponnaiyan, \\ L. Smart, D. Trbojevic, and S.Y. Zhang. \\ BNL, Upton, NY - 11973, USA
}

January 29, 2004

\begin{abstract}
In the Run-2 (2001), an unexpected vacuum pressure was observed with increasing currents in both gold and proton operation at RHIC. This pressure increase due to molecular desorption is suspected to be induced mainly by electron multipacting, but other causes may coexist. In order to obtain a reliable diagnostic of the phenomenon, electron detectors have been designed, and finally installed in the RHIC ring. The use of solenoids as a possible cure for the phenomenon has also been evaluated. This report describes both instrumentation and measurements during Run-3 (2002/03) at RHIC.
\end{abstract}

\section{Introduction}

A pressure increase was observed during the Au-Au Run-2 (2001) at the Relativistic Heavy Ion Collider (RHIC) for high intensity beams [1,2]. It is suspected to be due to molecular desorption, mainly sustained by electron multipacting, but other causes may exist such as ion desorption or beam losses (see Ref. [3]). In that case, the electron cloud $(E C)$ density was estimated from the measured coherent tune shift along the bunch train [4]. However, a more straightforward way to detect the $E C$ is the use of dedicated electron detectors $(E D)$. During the 2002 RHIC shutdown, $16 E D$ s were installed in the ring. A special RHIC ED was designed to measure the electron energy spectrum. Ion collection is also possible with this detector. EDs coming from SNS and ANL have also been used. Interested readers can find more information about them at [5] and [6].

*ubaldo@bnl.gov 
This report presents the design and calibration of the RHIC ED, and also discusses the results found with all the RHIC EC instrumentation during Run-3, which started in December 2002 and finished in May 2003.

\subsection{Electron Cloud instrumentation at RHIC}

The following $E C$ instrumentation was installed in RHIC for Run-3:

- Electron Detectors 11 RHIC EDs, 4 SNS EDs, 1 ANL ED, and 1 MicroChannel Plate (MCP) $E D$. The RHIC $E D$ will be fully explained in next section. The MCP has a large nominal gain $(G=58 \mathrm{~dB})$ to allow the detection of low electron fluxes.

- Pin Diodes. Devoted to detect beam halo scrapping, this instrumentation was not available during Run-3 due to technical difficulties.

- Solenoids. As a possible cure to $E C$, solenoids were wrapped along about a $7 \%(64 \mathrm{~m})$ of the warm beam pipes. The maximum achievable magnetic field is $68 \mathrm{G}$.

- Vacuum instrumentation. Several vacuum gauges, including a Residual Gas Analyzer $(R G A)$ allow pressure rise studies.

All this instrumentation is devoted to analyze the $E C$ at RHIC, and it was installed at the interaction regions labelled 12 and 2 (IR12, and IR2), and adjacent straight sections. See Fig. 1 for a drawing of the locations where the $E D$ instrumentation were installed.

\section{The RHIC electron detector}

The working principle of the RHIC ED is similar to the one described in Ref. [7]. Its layout can be seen in Fig. 2. The top grid ('Grid 0' in Fig. 2) acts as an RF shield, and its transparency $\left(T_{0}\right)$ is fixed to $23 \%$ in order to decrease the effect of the image currents without interfering with the multipacting process. The middle grid (stated in Fig. 2 as 'Grid 1', with $T_{1}=80 \%$ ) can be biased to different voltages through the remote controlled High Voltage supply $(H V)$. It acts as an energy filter, allowing the electron energy $\left(E_{e}\right)$ spectrum to be measured. The bottom grid ('Grid 2' in Fig. 2, with $T_{2}=80 \%$ ) is held at $-10 \mathrm{~V}$ by a DC battery. It is used to repel back the secondary electrons produced at the collector. The collector can be biased either positively or negatively through another output of the HV supply in order to check the presence of either electrons or ions, respectively [3].

In order to be able to keep the collector at different voltages and polarities without damaging the amplifier, an $R L C$ circuit (Fig. 2) is used in a box placed as close as possible to the ED in the ring. The multiplexer (shown as 'MUX' 


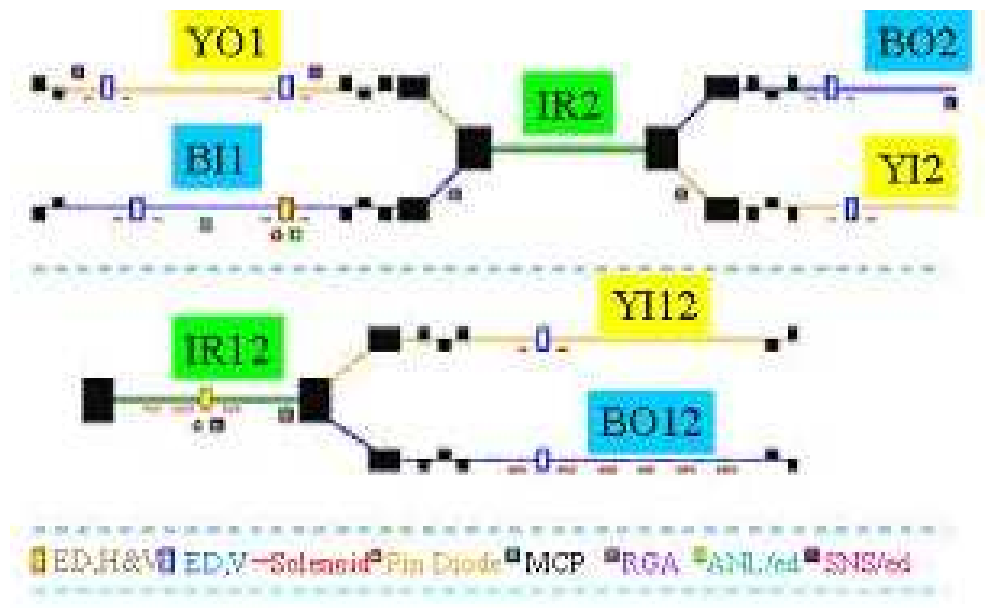

Figure 1: Electron detection instrumentation installed in RHIC warm sections in interactions regions 12 and 2 .

in Fig. 2) allows for signal splitting and two data acquisition modes: 'fast' and 'slow'.

The fast mode uses a scope to take a $20 \mu$ s snapshot sampling at $1 \mathrm{GHz}$ which is done through the scope. This acquisition is triggered every AGS ${ }^{1}$ cycle (approximately every $4 \mathrm{~s}$ ). This provides information about the build and decay time of the cloud within a turn (i.e., $12.8 \mu \mathrm{s}$ ), as well as the saturation value for the flux (or current) into the wall $\left(I_{\text {wall }}\right)$.

The 'slow' mode uses a Multiplex Analog to Digital Converter (MADC) with a sampling rate of $720 \mathrm{~Hz}$. This mode should be useful in linking the time evolution of pressure $(P)$ and electron flux into the wall for large time scales (minutes).

\subsection{RHIC ED Calibration}

For a given scope voltage reading $(V)$, we are interested in the corresponding electron flux, or current, into the wall: $I_{\text {wall }}$. Given the surface area of the detector $\left(S_{e d}=78 \mathrm{~cm}^{2}\right), I_{\text {wall }}$ in terms of $\mathrm{A} / \mathrm{cm}^{2}$ can be calculated as:

$$
I_{\text {wall }}=\frac{V}{Z G S_{\text {ed }} T_{\text {eff }}},
$$

where $Z=50 \Omega$, and $G$ is the nominal gain of the amplifier, which is significantly flat for the frequency range we are interested in $(80 \mathrm{kHz}$ to $20 \mathrm{MHz})$.

\footnotetext{
${ }^{1}$ Alternating Gradient Synchrotron
} 


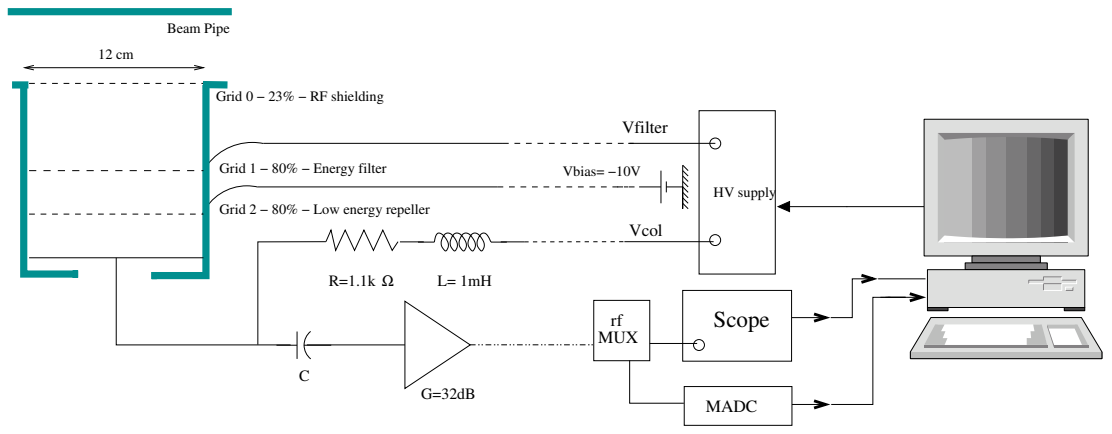

Figure 2: Layout of the RHIC ED. The capacitance $C$ is not the same for all 11 detectors, but a typical value is $0.01 \mu F$. The capacitance $C$ is needed to protect the amplifier.

Although one can estimate the effective transparency $\left(T_{\text {eff }}\right)$ as $T_{\text {eff }}=T_{0} \cdot T_{1} \cdot T_{2}$, this ideal relation does not take into account the electron energy: low energy electrons have larger probability to be lost through the grids 0,1 , and 2 (see Fig. 2) than high energy ones. This effect is calibrated in Fig. 3, which shows the effective transparency $\left(T_{e f f}\right)$ of the $E D$ as a function of the voltage applied to grid 1 ( $\left.V_{\text {filter }}\right)$, which represents the electron energy. The electron energy spectrum of the $E C$ in RHIC (see below) shows very low energy electrons, from which one can estimate that the average $T_{\text {eff }}=5 \pm 1 \%$. Therefore, the uncertainty in $T_{\text {eff }}$ will be responsible for the error in computing the flux into the wall from the experimental data $(V)$. More details about the calibration process can be found at Ref. [5].

\subsection{Signal differentiation}

An AC coupled system differentiates the signals below the low frequency cutoff $\left(f_{\text {low }}\right)$, which is determined by the capacitance, $C$. A typical value of $C$ is $0.01 \mu F$, which corresponds to a $f_{\text {low }}=2 M H z$. The RHIC $E D$ circuit has been evaluated using the commercial software PSPICE ${ }^{2}$, and its behavior has been tested for a given 'ideal' current into the wall. This current has been taken from one of the existing computer $E C$ simulation codes, in this case, $C S E C$ (see Ref. $[4,11]$ ). Figure 4 shows the signal differentiation when the EC starts. Although we are referring to an electron current which should always be negative (green light points in Fig. 4), the signal that will be seen in the scope (black line) is both positive and negative in order to keep the integral over one period null. In this case, the period is the RHIC revolution time: $\tau_{\text {rev }}=12.8 \mu \mathrm{s}$. We can see that as the $E C$ takes place, both the maximum and minimum parts of

\footnotetext{
${ }^{2}$ http://www.orcadpcb.com/pspice
} 


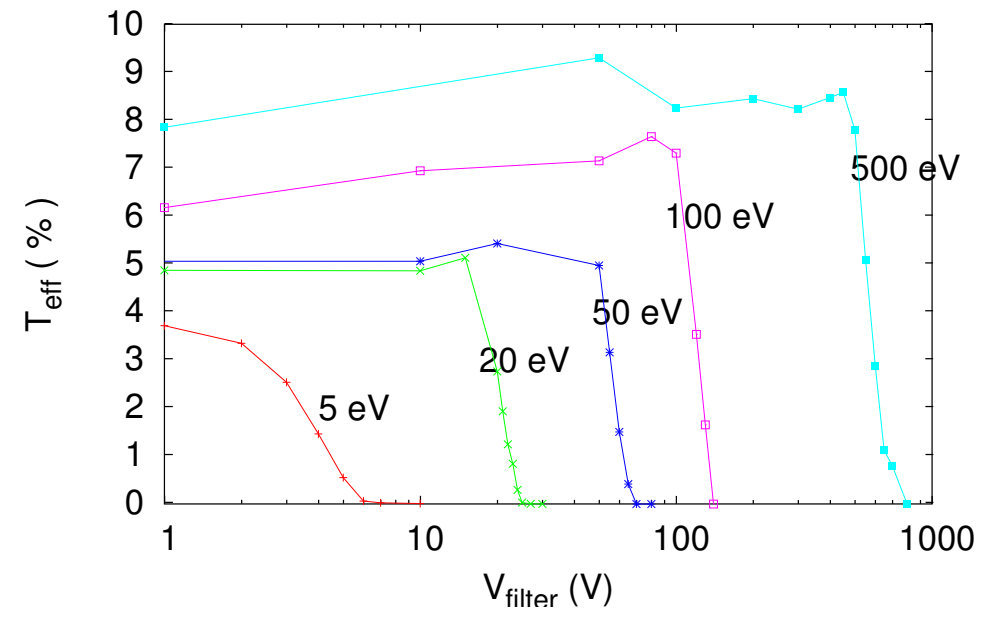

Figure 3: Effective transparency of the $E D$ as a function of $V_{\text {filter }}$. Note the logarithmic scale in the horizontal axis.

the signal change in magnitude due to the electronics design. Using the MADC, we $\log$ the maximum and minimum values of the 720 samples taken per second and we can follow the slow evolution of $E C$ at $1 \mathrm{~Hz}$. This will become the most reliable tool in evaluating the $E C$ signal at large time scales (minutes).

\section{Experimental results}

During Run-3, two kinds of collisions were scheduled for RHIC. Deuterons (d) were colliding with gold ions $(\mathrm{Au})$ from December 2002 until late March 2003. Polarized protons (p) were run from then until late May 2003.

In $d A u$ operation, $\mathrm{d}$ circulated in the clockwise RHIC ring (blue ring), and the $A u^{79+}$ ions ran in the counterclockwise RHIC ring (yellow ring). Figure 5 shows the chronological evolution of both total and per bunch intensities for the $d A u$ Run-3. Note the intensity per bunch is below the $E C$ conditions in Run-2 (see Table 1 and Ref.[1]). Although $d$ bunch intensity exceeded $10^{11}$ later in the run, only occasionally this intensity reached the $E C$ threshold for $\mathrm{Au}$. Together with the bake out, these facts did not produce severe pressure rises (see [8] for more details), and the possibilities of detecting electrons were significantly reduced. More information about technical issues of Run-3 can be found at Ref. [9].

On the other hand, clear $E C$ cases occurred during the $p p$ operation, where it was technically feasible to reach higher intensities per bunch than during the $d A u$ operation. These cases were logged mainly by the $E D$ placed at the long 


\section{Signal differentiation}

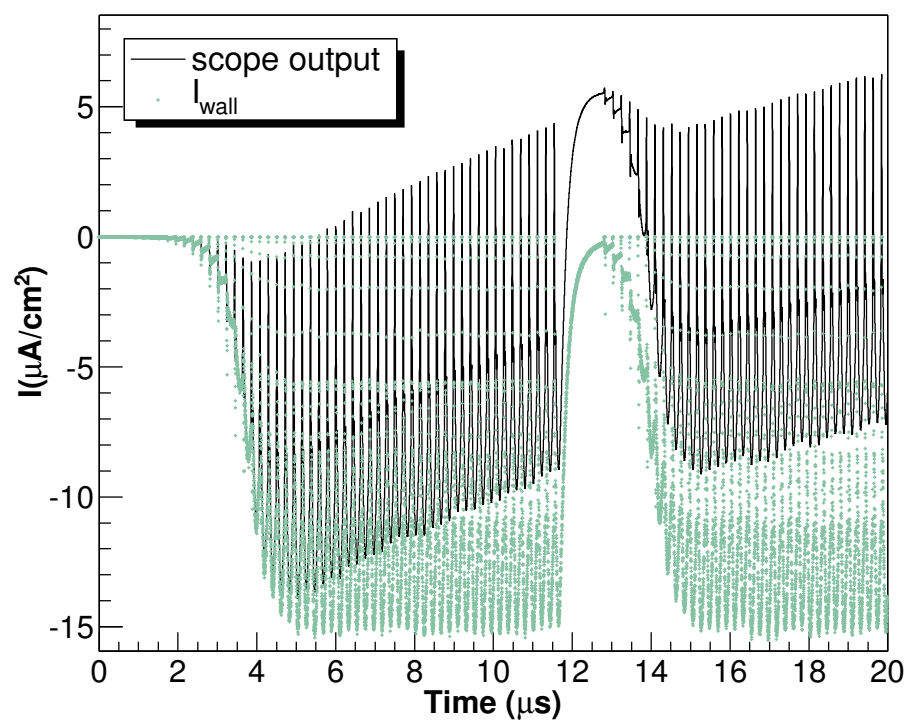

Figure 4: $I_{\text {wall }}$ calculated with $C S E C$ (green light dots). This signal is differentiated by the RHIC ED system, and has been evaluated with PSPICE (black line).

straight section labeled 'bo2', where the vacuum chamber has cylindrical symmetry and its surface type is unbaked stainless steel.

As explained in [10], we use in the following triplets of integer numbers $\left(k_{s}, k_{b}, k_{g}\right)$ to describe bunch patterns: $k_{s}$ gives the bunch spacing in buckets, $k_{b}$ the number of bunches filled with that spacing, and $k_{g}$ the number of "phantom" bunches added, i.e. bunches that are not filled in and therefore create a gap. For example the configuration $(2,2,1)(3,4,0)$ would correspond to the pattern

$$
\text { 1-0-1-0-0-0-1-0-0-1-0-0-1-0-0-1-0-0 }
$$

where 1 denotes a filled and 0 denotes an empty bucket. If not otherwise noted, it is assumed that a pattern repeats until the abort gap is reached. Since a bucket in RHIC corresponds to $35.6 \mathrm{~ns}$, the bunch spacing in ns can be expressed as $35.6 \times k_{s}$.

In this section we show the $E C$ evidences during Run-3 for the three species: $\mathrm{d}, \mathrm{Au}$, and p. Table 1 summarizes the main beam characteristics of the fills studied in this report. 


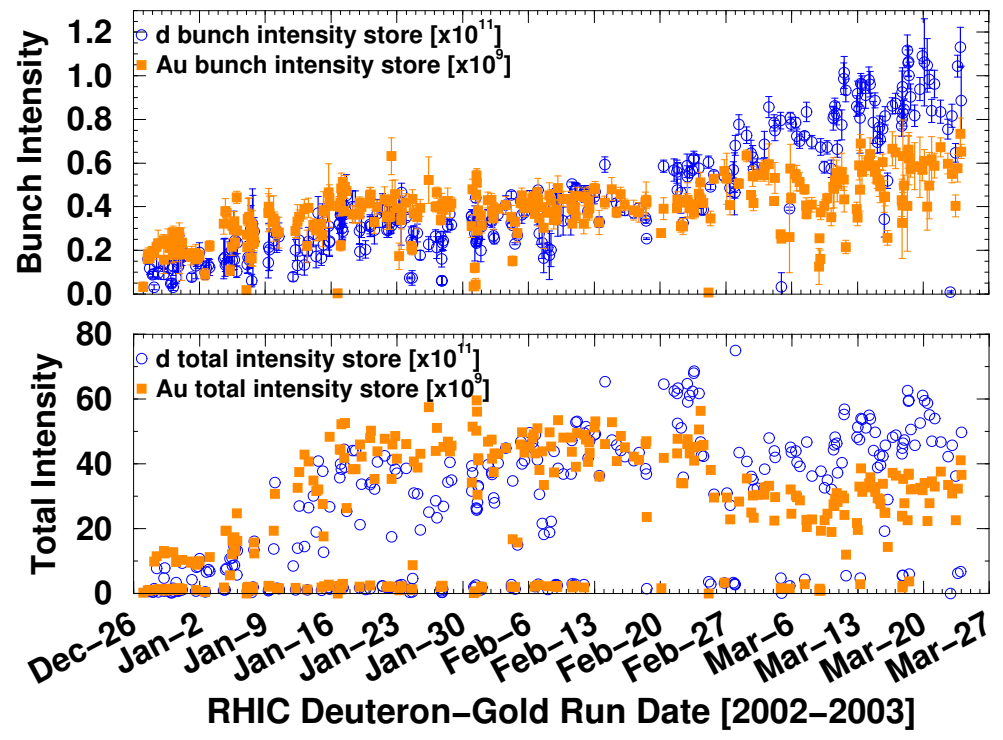

Figure 5: Evolution of RHIC dAu total and per bunch intensities measured at the end of every acceleration ramp (unseccesful ramps are excluded). From 9 January to 27 February, RHIC was operated with 110 bunches per ring, otherwise with 56 bunches per ring. This is why the increasing per bunch intensity does not corresponds with increasing total intensities from February 27th. Courtesy of T. Satogata.

\subsection{The Gold case}

The only case reported with a clear EC signal in the Au ring is shown on Fig. 6 . The bunch intensity was pushed up to $I_{p b}=7.5 \cdot 10^{8} A u p b$ in a bunch pattern $(3,110,10)$ : a train of 110 bunches and $107 \mathrm{~ns}$ bunch spacing. A pressure rise suspected to be due to $E C$ is detected during the injection process, but no signal was logged at that time $(6 \mathrm{~h} 09 \mathrm{~m})$. Around $6 \mathrm{~h} 12 \mathrm{~m}$, a small 'transition type' pressure rise is detected when the ramping process started (see Ref. [8] for details about this kind of pressure rise), and as the bunches were getting shorter, a strong $E C$ was triggered few seconds later. See Fig. 7 for a snapshot of the $E C$ evidence. Within 10 seconds the vacuum pumps (ion pumps) saturated and automatically switched off, what finally lead to $P \approx 2 \times 10^{-3}$ Torr and to the loss of the beam.

Figure 7 shows the signal in the $E D$ for that region (labelled as 'yo1') and for that particular case. This snapshot is taken only $6 \mathrm{~s}$ before the ion pumps switched off. As shown in the PSPICE simulations and because of the AC coupling $E D$ design, once the phenomenon is triggered, the voltage $V$ goes 


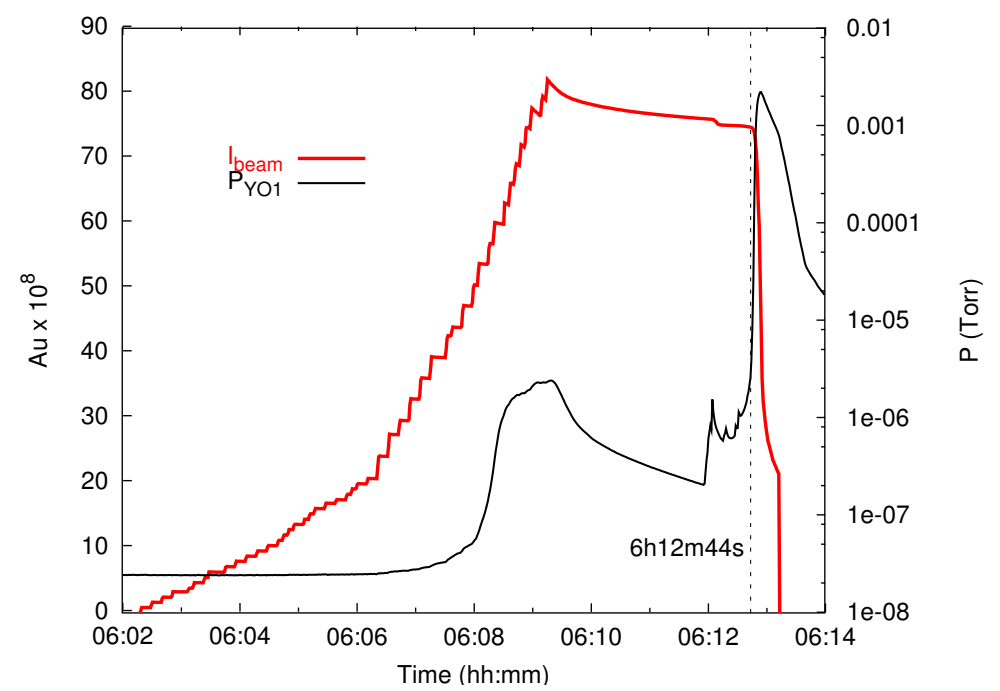

Figure 6: Evolution of the beam intensity in the yellow ring and pressure in section 'yo1' for fill \#3107. Section 'yo1' was unbaked stainless steel.

from positive to negative values as the bunches are passing by, and turn back to the initial positive value. Unfortunately, the signal was so high that the scope saturated at $\pm 0.04 \mathrm{~V}$. According to the detector's calibration (see Fig. 3), the corresponding current into the wall is about $5 \mu \mathrm{A} / \mathrm{cm}^{2}$. Note how a lower per bunch intensity produces a decay in the multipacting signal. The decay between bunches 55 to 58 is specially noticeable. Together with the previous experience (no $E C$ signal was logged for similar beams but with $I_{p b}=5.5 \times 10^{8} \mathrm{Aupb}$ ) this might indicate that the threshold at transition for $E C$ in unbaked stainless steel surfaces at RHIC should range between $5.5 \times 10^{8} \mathrm{Aupb}$ (the lower intensity per bunch in Fig. 7) and $7.5 \times 10^{8} \mathrm{Aupb}$. For other vacuum pipes surfaces in the machine, this threshold is not valid, and higher intensities per bunch are needed to trigger the effect. High intensities were not readily attainable for Au during Run-3 (see Fig. 5 and Ref. [9]), and this is the only case reported. This case was the first direct measurement of electron clouds at RHIC.

\subsection{The deuteron case}

In the d case, no clear snapshots of data have been logged with the $E D$. However, in some cases by smoothing the data it is possible to distinguish the $E C$ signal from the noise level and image currents. The most critical region in the blue ring turned out to be the straight section 'bo2'. The RHIC ED there has the same characteristics as seen in Fig. 2, except that it is equipped with 2 am- 

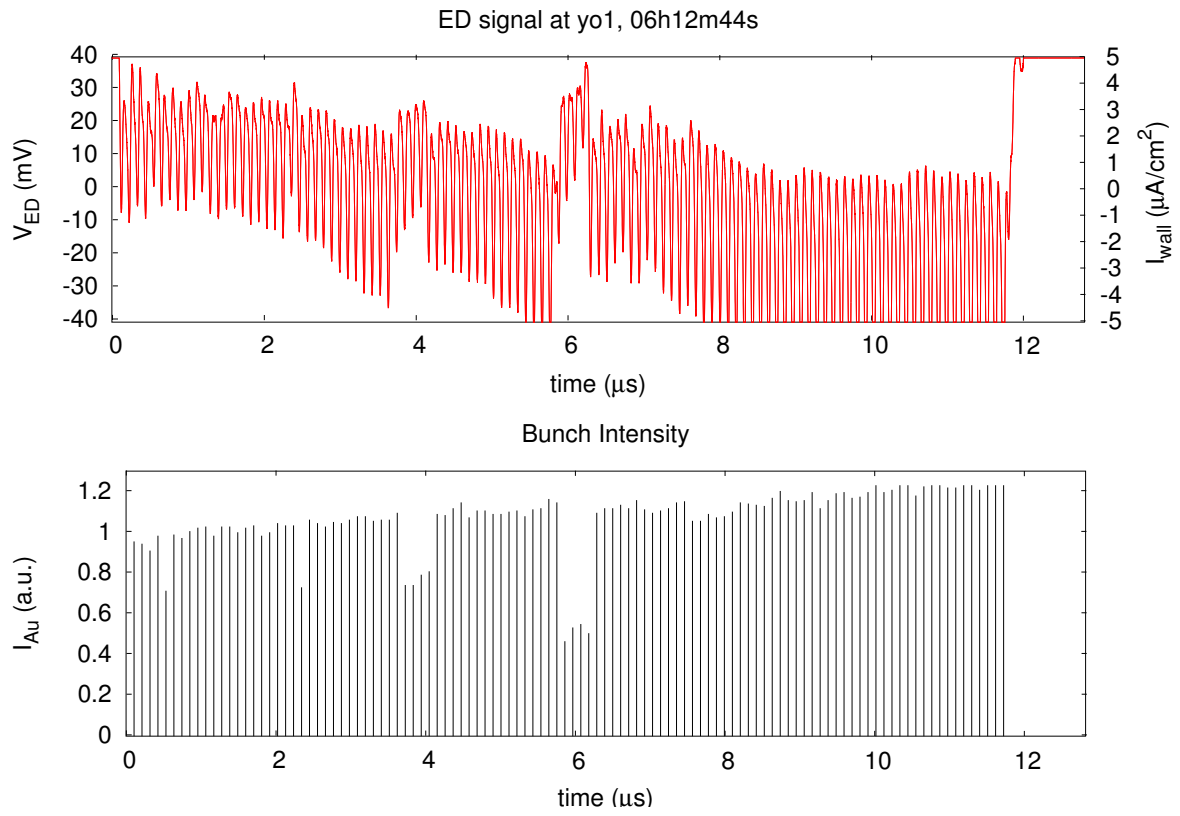

Figure 7: $E C$ signal on the $E D$ placed in 'yo1' sector. The snapshot on the top shows the evolution of the cloud during a RHIC turn $(12.8 \mu \mathrm{s})$, whereas the bottom one shows the bunch structure for fill \#3107. Note the decay in the signal for bunches with lower intensity, specially from bunches 55 to 58 . The snapshot is taken at 06:12:44, when the pressure is $2.6 \cdot 10^{-6}$ Torr.

plifiers in series. Therefore its total nominal gain is $G=64 d B$. Fig. 8 reports the case of fill \#3159, whose characteristics can be seen in Table 1. When 59 bunches were injected in the machine, the raw data does not show any clear $E C$ signal (light green dotted lines). Numerically smoothing the data with a $10 \mathrm{MHz}$ low-pass filter (black line), we can clearly see how the $E C$ rises for the last bunches and disappears at the same time as the bunch train. The pressure rised from $10^{-8}$ to more than $10^{-6}$ Torr.

\subsection{The polarized protons run}

The $E D$ placed in 'bo2' showed clear $E C$ signals during the $p p$ run using the 'smoothing' technique, and similar snapshots as for the $d$ case are shown in Fig. 9 for fill \#3460 (see Table 1 for beam characteristics of this fills). Again, one can see that both the $10 \mathrm{MHz}$ filter (bottom trace) and the $20 \mathrm{MHz}$ filter (middle trace) are useful to distinguish the signal from the raw data (top trace). Since 


\section{Raw data and smoothed signal. Fill \#3159, 8h59m16s}

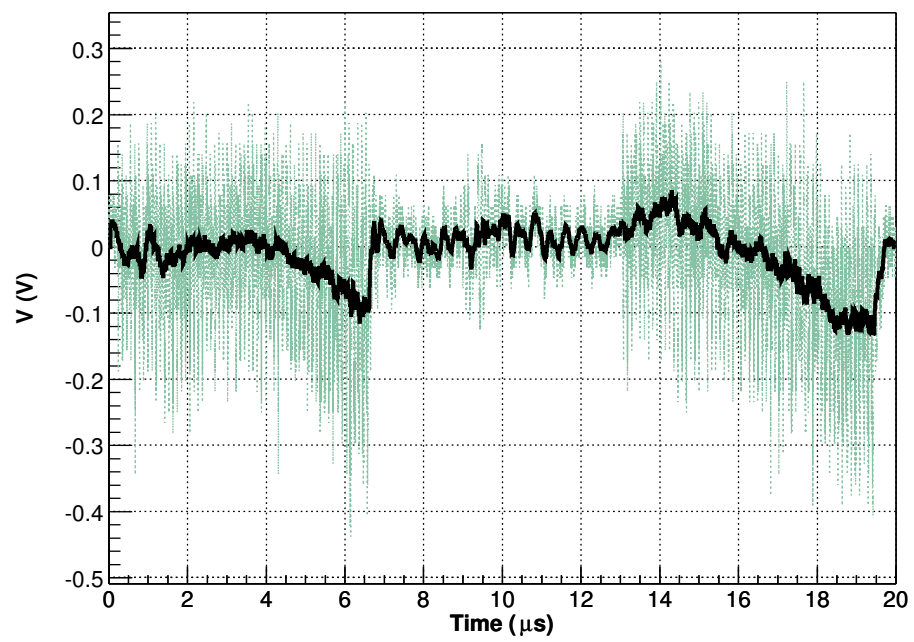

Figure 8: $E C$ signal on the $E D$ placed in the 'bo2' sector. The light dotted green line shows the raw data, whereas the black solid line shows the smoothed data using a numerical $10 \mathrm{MHz}$ filter. The pressure at the moment the snapshot is taken is $4.1 \cdot 10^{-7}$ Torr.

the scope ${ }^{3}$ used in the detection (see Fig. 2) has the 20MHz smoothing option, this feature was implemented in the data collection from then on (mid May).

\subsubsection{Pressure rise and electron flux into the wall}

Fill \#3460 (see Table 1) showed a general EC problem throughout RHIC. This clear case allowed us to carry out interesting studies using the 'slow' mode (MADC). The bottom plot in Fig. 10 shows the time evolution of the pressure, and the signal in the $E D$. The injection during this fill is shown in the top plot in Fig. 10. Injection was temporally interrupted when 45 bunches were injected $(\mathrm{t}=250 \mathrm{~s})$, it resumed at $\mathrm{t}=320 \mathrm{~s}$ and finished after 700s. Correlation between the time evolution of pressure and electron signal was visible. Although the pressure starts to rise about 100 s earlier, at $t=200$ s the electron signal indicates an electron avalanche due to multipacting and the pressure rises rapidly, which shows that $E C$ is the primary factor triggering the pressure rise. Using Eq. 1 and the calibration in Fig. 3, one estimate that the electron flux into the wall in this plot ranges from 0.5 to $2 \mu \mathrm{A} / \mathrm{cm}^{2}$.

\footnotetext{
${ }^{3}$ Waverunner 2 LT354-M, 1GS/s.
} 

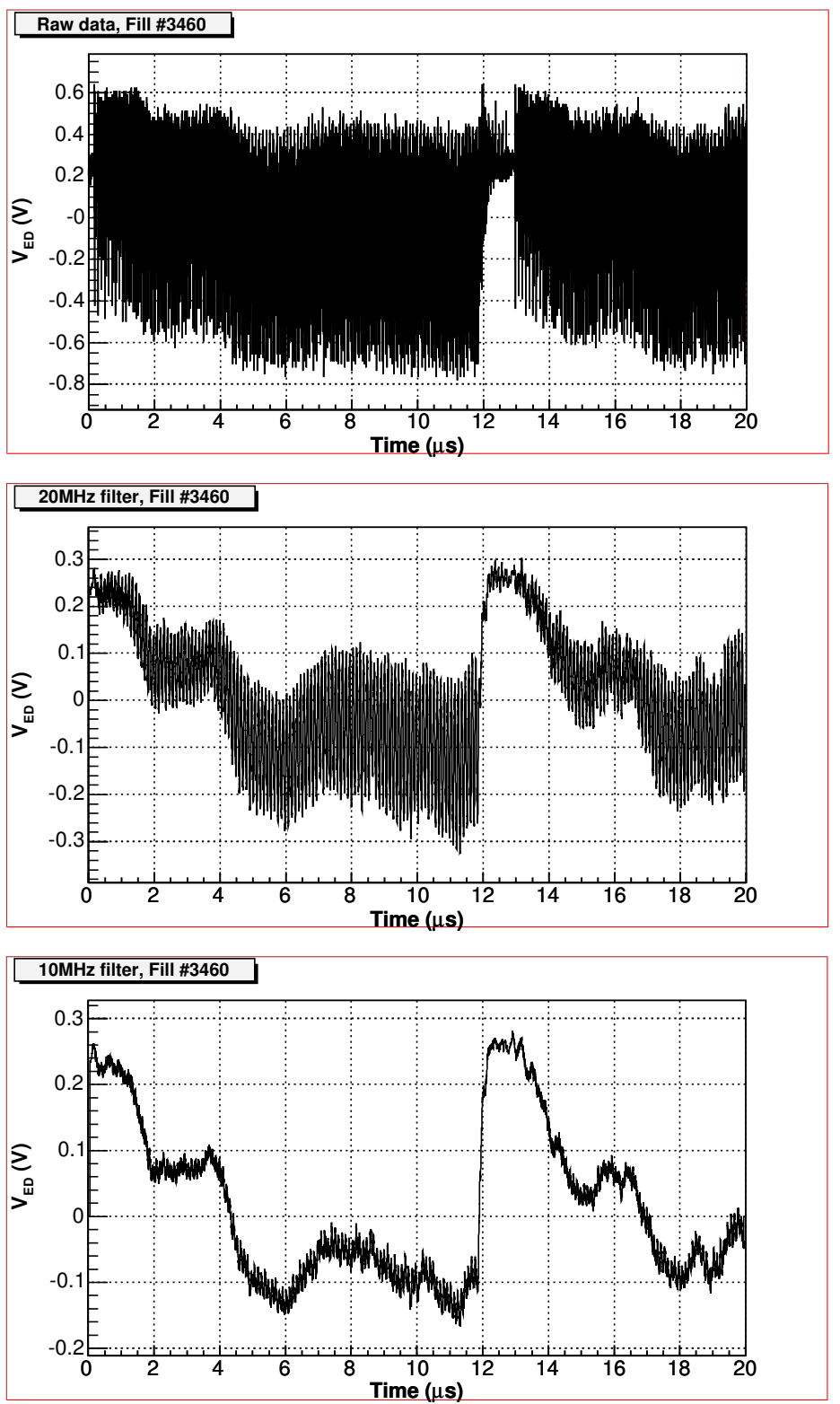

Figure 9: $E C$ signal using the fast mode observation. We can see that the raw data do not show any clear signal, but after applying a 10 and $20 \mathrm{MHz}$ filter, the $E C$ signal shows up clearly. This snapshot is taken when during fill \#3460, when 110 bunches were injected in the machine and the pressure gauge showed $3.6 \cdot 10^{-6}$ Torr. 

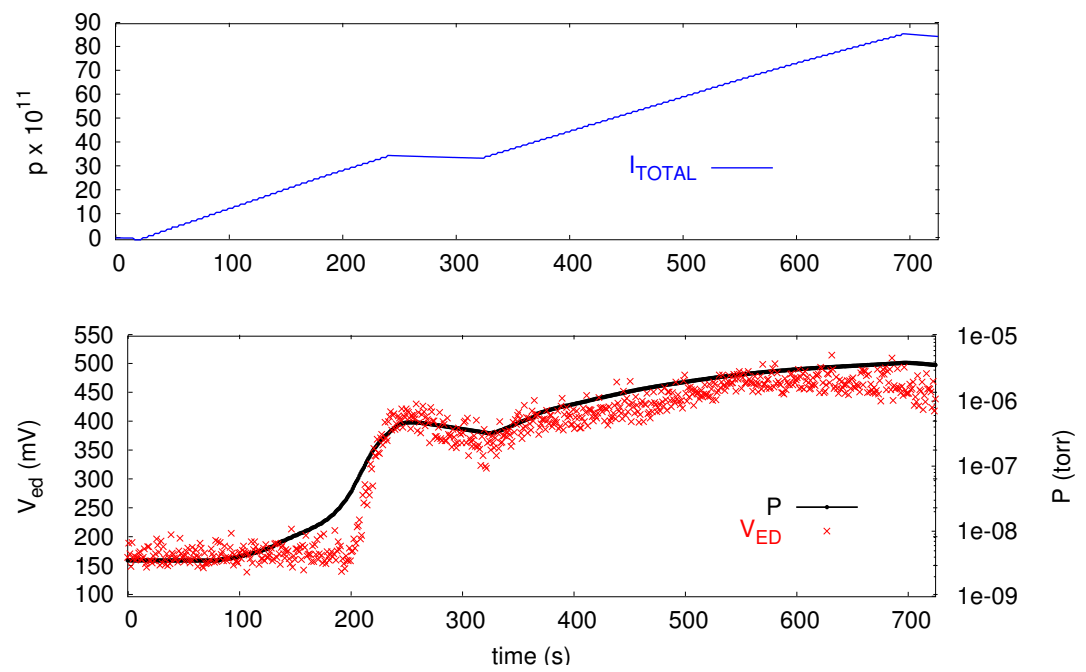

Figure 10: The top figure shows the total beam current injected in RHIC. Note that injection was temporally interrupted after 45 bunches $(t=200 \mathrm{~s})$ and resumed at $\mathrm{t}=320 \mathrm{~s}$. The bottom figure, shows the pressure evolution (black line) and $E C$ signal using MADC (red crosses) while this injection takes place. (Data taken in sector 'bo2').

\subsubsection{Electron energy spectrum}

A very important result for the EC understanding becomes the electron energy spectrum. In the RHIC case, the logging of this electron energy spectrum was done during fill \#3812 (see Table 1) using the equipment shown in Fig. 2. Figure 11 shows an integrated electron energy spectrum, this is the signal collected in the ED placed at 'bo2' when sweeping $V_{\text {filter }}$ from 0 to $500 \mathrm{~V}$. These two sweeps were done when only 39 bunches (bunch spacing is $107 \mathrm{~ns}$ ) were running at RHIC. The two main aspects to stress is first the large peak of low energy electrons, and second how the spectrum extends to about $300 \mathrm{eV}$. Note that the noise in the ED is around $150 \mathrm{mV}$. Therefore, we cannot definetely conclude the upper energy limit for the electron. However, using the fast mode observation, we were able to distinguish electrons up to about $500 \mathrm{eV}$. It is worth mentioning that the intensity per bunch for this fill was unusually high (up to $2.0 \cdot 10^{11} \mathrm{ppb}$ ). See Fig. 12 and Table 1 for more details about this bunch pattern. 


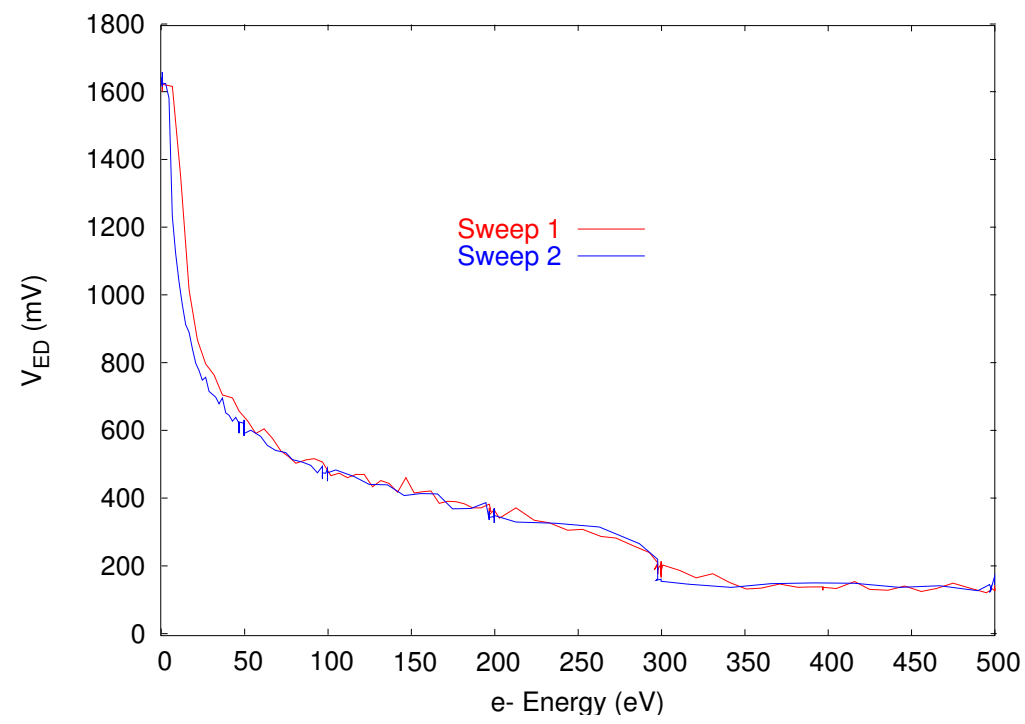

Figure 11: Electron signal in the $E D$ when filtering the electron energy from 0 to $500 \mathrm{~V}$. This corresponds to an integrated energy spectrum.

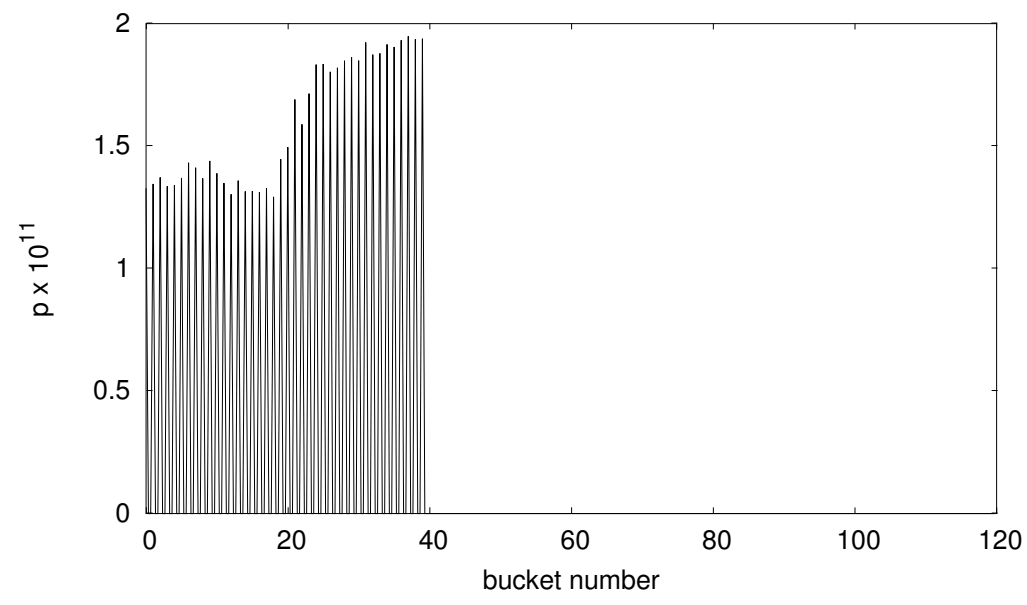

Figure 12: Bunch intensity for the bunches injected at RHIC when the $V_{\text {filter }}$ sweep was done. 


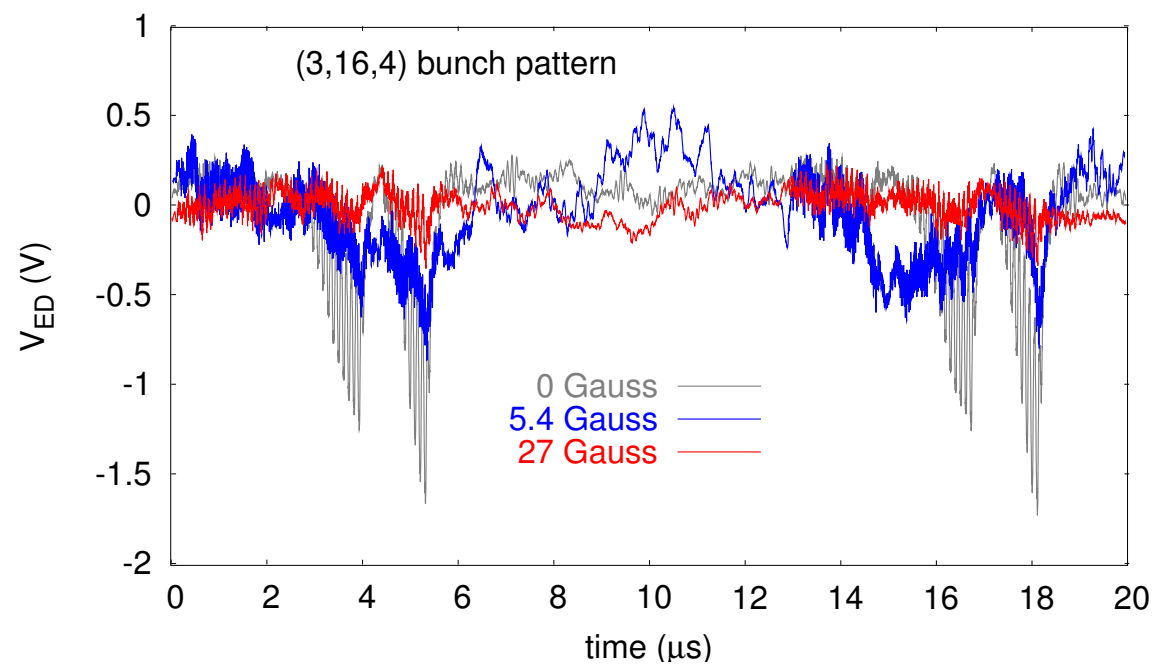

Figure 13: Results of applying different solenoid fields to suppress the $E C$. For $B_{\text {sol }}=27$ Gauss, the signal is already below the noise level of the $E D$.

\section{Possible cures}

\subsection{Solenoid field results}

One of the solutions thought to suppress the $E C$ was the use of solenoids. Such a method has been successfully tested in other laboratories (see the PSR experience in Ref. [14]). In the RHIC case, further studies will be necessary in order to understand the problem. While results from fill \#3530 indicate that solenoid fields $\left(B_{\text {sol }}\right)$ can help to fully suppress the cloud (see Fig. 13), this is not certainly true for fill \#3812. Figure 14 shows how the electron signal is not completely suppressed even though we reached the maximum solenoid field $\left(B_{s o l}=68\right.$ Gauss $)$. Note, as pointed out earlier, that the intensity per bunch is unusually high for fill \#3812 (see beam characteristics of this fill in Table 1). But the question is not only whether the magnitude of $B_{\text {sol }}$ is high enough to suppress the cloud for any intensity per bunch. Fill \#3667 shows an interesting effect: some values of $B_{\text {sol }}$ increased the pressure, instead of decreasing it (see Fig. 15). As explained in Ref. [12], this can be due to resonance effects between the cyclotron frequency of the multipacting electrons and bunch spacing, but the RHIC parameters do not fit this explanation. Also, the direction of the solenoid field plays a significant role (see Ref. [12]), and this case is currently being studied using POSINST code [13]. 


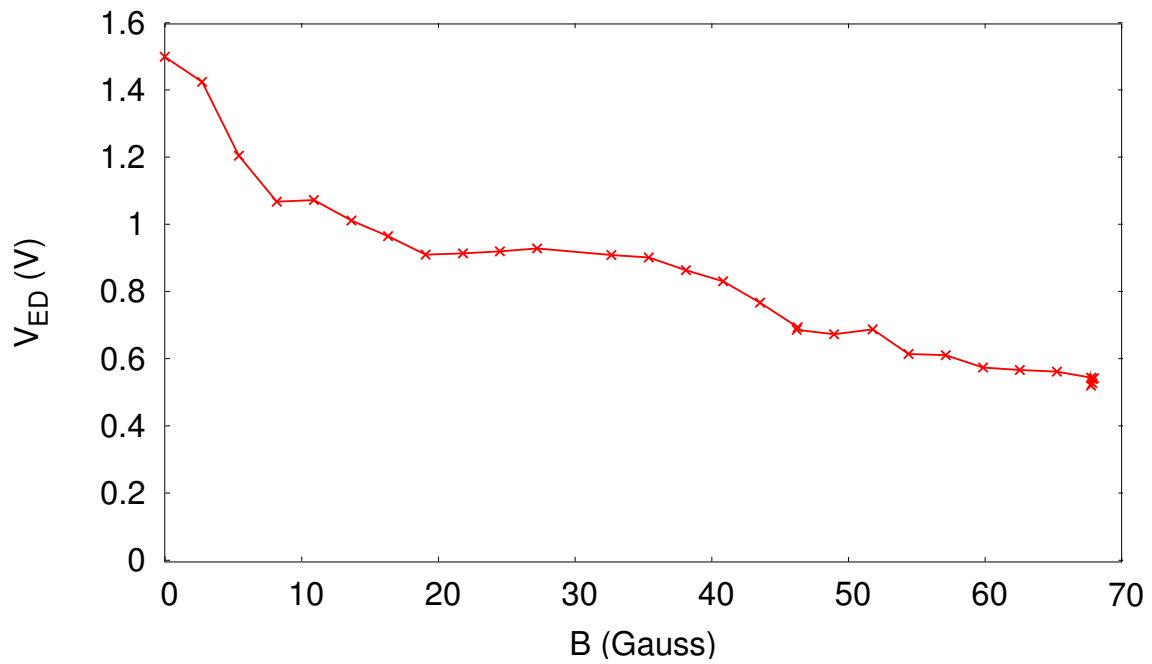

Figure 14: Electron signal while sweeping the solenoid field from 0 to 68 Gauss. Even at the maximum available field, the multipacting is not fully suppressed.

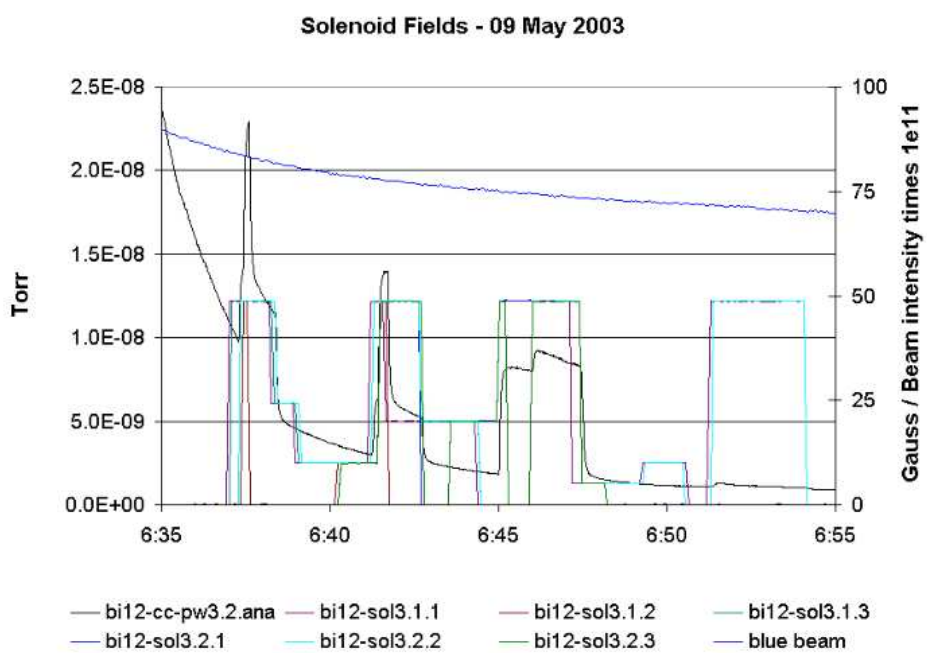

Figure 15: Pressure time evolution for different values of the solenoid field. Note how the pressure grows for certain values of $B_{\text {sol }}$. 

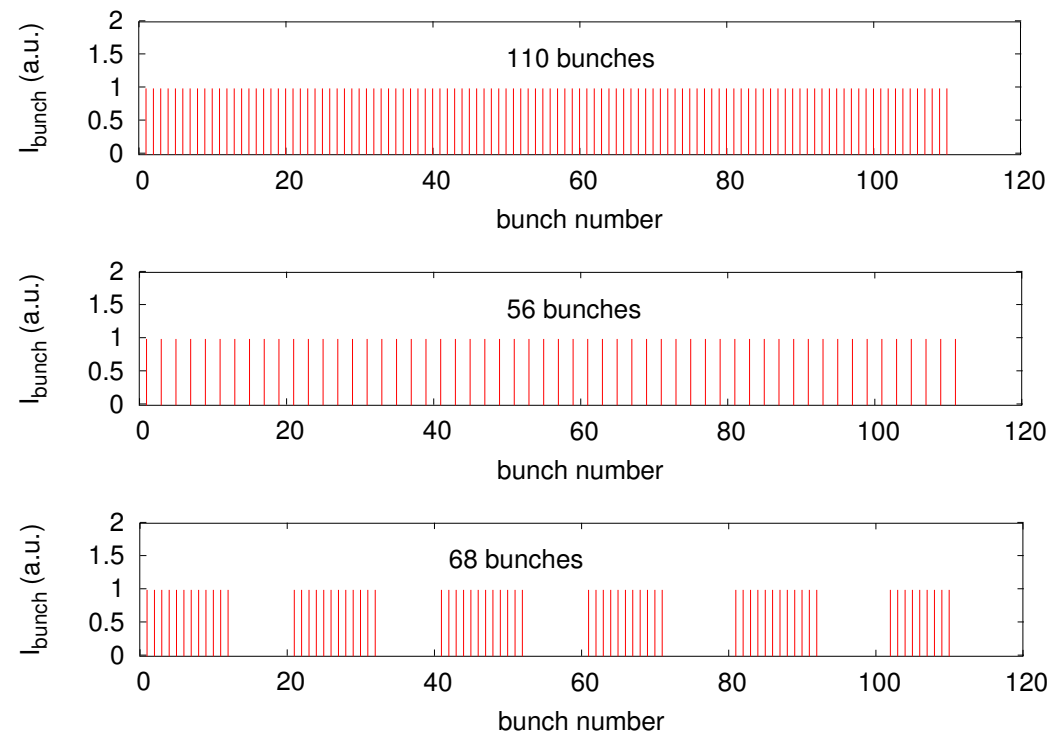

Figure 16: Three different bunch patterns used in RHIC during Run-3. The top one $(3,110,10)$ maximizes the luminosity in RHIC, but also creates the most severe electron clouds. The middle one $(6,56,4)$ corresponds to the nominal conditions, and did not show EC during Run-3. The bottom one $(3,12,8)$ allows 68 bunches in the machine, which still provides $20 \%$ more Luminosity than the middle one.

\subsection{Missing bunches study}

Another method to suppress the $E C$ is to leave out bunches in the bunch train. Since the growth time is longer than the decay time (see experimental observations in Figures 9, and 13), one could find a pattern which does not trigger the effect. Of course, we lose some Luminosity $(L)$ because we did not inject the maximum number of bunches for a bunch train, but we still improve the initial nominal performances. In other words, although it may still not be possible to run with 110 bunches in the next run, it may be possible to run with more than 56 bunches. With RHIC's six-fold symmetry, the bunch pattern $\left(k_{s}, k_{b}, k_{g}\right)$ must have a three-fold symmetry to have approximately the same number of collision in all experiments. Due to the abort gap some experiments have about $10 \%$ less bunch-bunch collisions than other experiments.

On 23 April 2003 three different bunch patterns were tested: $(3,16,4),(3,12,8)$ and $(3,14,6)$. These cases are compared in detail at [10]. Experimental observations in the $E D$ for $(3,16,4)$ and $(3,12,8)$ can be seen in Figures 13 , and 17 . These bunch patterns still increase Luminosity with respect to the nominal running 

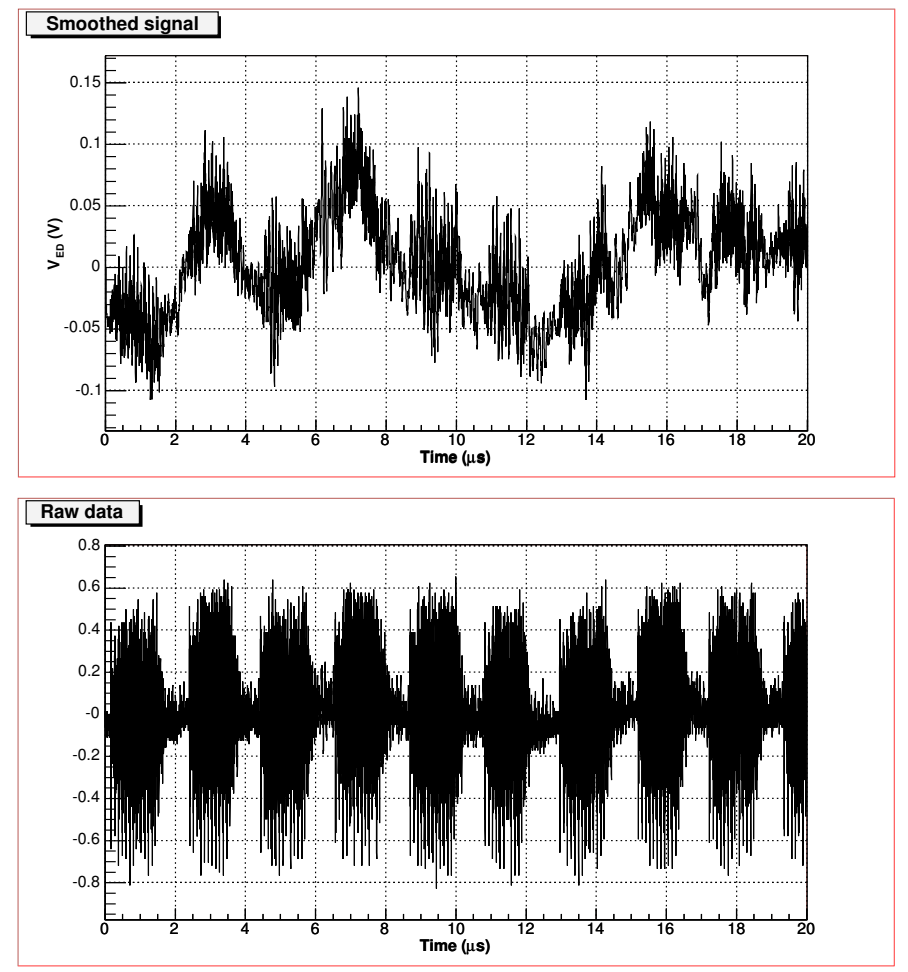

Figure 17: Raw ED data (bottom) and smoothed signal (top) when the $(3,12,8)$ bunch pattern configuration is in RHIC. Neither one shows any multipacting trace, but the effect of image currents and noise.

conditions $(6,56,4)$. In particular, the pattern $(3,12,8)$ succesfully minimizes the effects of the $E C$ : Fig. 17 shows only noise is collected for $I_{p b}=10^{11} \mathrm{ppb}$, and the pressure rise was not significant. This bunch pattern gives 68 bunches circulating in the ring, which still provides $20 \%$ more Luminosity than the nominal running conditions.

\section{Conclusions and outlook}

The EC at RHIC has been observed with d, Au, and p during the Run-3 using the RHIC ED. Due to the electronics design of the ED, the signal is differentiated. However, using PSPICE we can evaluate the signal differentiation and it is possible to estimate the evolution of $E C$ for large time scales (minutes). Nevertheless, some laboratory studies are currently being carried out to improve the RHIC ED performances, mainly focused on its transparency and a new DC 
Table 1: Main characteristics of fills suspected of leading to $E C$ during Run-2, compared with the Run-3 fills in this note. The average intensity and the total number of bunches refers to the moment where the $E C$ related measurements reported in this note were done.

\begin{tabular}{|c|c|c|c|c|}
\hline \multicolumn{5}{|c|}{ RUN-2 } \\
\hline Fill \# & $\begin{array}{l}\text { average bunch } \\
\text { intensity, } I_{p b}\end{array}$ & bunch pattern & $\begin{array}{l}\text { total number } \\
\text { of bunches }\end{array}$ & $\begin{array}{l}\text { bunch } \\
\text { spacing }\end{array}$ \\
\hline 1797 & $9.0 \cdot 10^{8} A u^{79+}$ & $(6,56,4)$ & 56 & $214 \mathrm{~ns}$ \\
\hline 1798 & $7.5 \cdot 10^{8} A u^{79+}$ & $(3,110,10)$ & 110 & 107 ns \\
\hline 2189 & $1.1 \cdot 10^{11} \mathrm{p}$ & $(6,56,4)$ & 56 & $214 \mathrm{~ns}$ \\
\hline 2237 & $0.8 \cdot 10^{11} \mathrm{p}$ & $(3,110,10)$ & 110 & $107 \mathrm{~ns}$ \\
\hline \multicolumn{5}{|c|}{ RUN-3 } \\
\hline Fill \# & $\begin{array}{l}\text { average bunch } \\
\text { intensity, } I_{p b}\end{array}$ & bunch pattern & $\begin{array}{l}\text { total number } \\
\text { of bunches }\end{array}$ & $\begin{array}{l}\text { bunch } \\
\text { spacing }\end{array}$ \\
\hline 3107 & $7.5 \cdot 10^{8} A u^{79+}$ & $(3,110,10)$ & 110 & $107 \mathrm{~ns}$ \\
\hline 3159 & $0.9 \cdot 10^{11} \mathrm{~d}$ & $(3,110,10)$ & 110 & $107 \mathrm{~ns}$ \\
\hline 3460 & $1 \cdot 10^{11} \mathrm{p}$ & $(3,110,10)$ & 110 & $107 \mathrm{~ns}$ \\
\hline 3530 & $1.1 \cdot 10^{11} \mathrm{p}$ & $(3,16,4)$ & 41 & $107 \mathrm{~ns}$ \\
\hline 3530 & $1 \cdot 10^{11} \mathrm{p}$ & $(3,12,8)$ & 68 & 107 ns \\
\hline 3667 & $0.9 \cdot 10^{11} \mathrm{p}$ & $(3,110,10)$ & 110 & $107 \mathrm{~ns}$ \\
\hline 3812 & $1.5 \cdot 10^{11} \mathrm{p}$ & $(3,110,10)$ & 39 & $107 \mathrm{~ns}$ \\
\hline
\end{tabular}

amplifier [15]).

Due to the bake out performed during the 2002 shutdown and the technical difficulties in achieving high intensities per bunch, the pressure rises during the $d A u$ run have in general been lower than the run before. On the other hand, high intensities per bunch were achieved during the $p p$ run, and this fact allowed interesting studies. Using the slow mode detection, a direct link between the electron flux into the wall and the pressure could be established. The electron energy spectrum shows a large peak of low energy electrons $(<40 \mathrm{eV})$, and how the tail lasts up to $300 \mathrm{eV}$ (with no significant population).

Solenoid magnetic fields have been proved to be effective to decrease the multipacting effect, but it has also been observed that not even the maximum achievable solenoid field can fully suppress the problem (if the the intensity per bunch is high enough). New solenoids using kapton wires (wires allowing the baking process) are going to be used.

Another method to reduce the $E C$ has been the 'missing bunches' studies. In particular, the bunch pattern $(3,12,8)$ has shown positive results for the RHIC case. Further studies (see Ref. [10]) are currently being carried out to find the best bunch pattern configuration to minimize the multipacting problem. 
The solution to avoid the EC problem in RHIC is not yet achieved. The reduction of the Secondary Emission Yield of the beam pipe surface wall using NEG coatings (Non Evaporable Getter) is one of the most effective (and expensive) methods [16]. During Run-4, several experimental NEG coated beam pipes are going to be tested in RHIC.

\section{Acknowledgements}

The authors are thankful for discussions and tips with M. Blaskiewicz, H. Huang, S. Peggs, T. Roser, T. Satogata, P. Thieberger, and all the C-AD department for their support.

\section{References}

[1] H.C. Hseuh et al. "Analysis of beam induced pressure increases in RHIC warm vacuum sections". Proceedings of EPAC'02, Paris, 2002.

[2] S.Y. Zhang. "RHIC Vacuum Pressure Bump". BNL C-A/AP/67 (2001).

[3] W. Fischer et al. "Vacuum pressure rise with intense ion beams in RHIC". Proceedings of EPAC'02, Paris (France) 2002.

[4] W. Fischer, M. Blaskiewizc et al. "Electron cloud measurements and simulations for the Brookhaven Relativistic Heavy Ion Collider". Phys. Rev. ST Accel. Beams 5, 124401 (2002).

[5] P. He et al. "Calibration of RHIC electron detectors". Proceedings of PAC'03. Portland, (USA) 2003.

[6] U. Iriso et al. "Electron detectors for vacuum pressure rise diagnostics at RHIC". Proceedings of PAC'03. Portland, (USA) 2003.

[7] R. Macek et al. "Recent experimental studies of the Electron Cloud at the Los Alamos PSR". KEK Workshop, Sept. 2001.

[8] S.Y. Zhang et al. "RHIC pressure rise and electron cloud". Proceedings of PAC'03. Portland, (USA) 2003.

[9] T. Satogata et al. "Comissioning of RHIC deuteron-gold collisions". Proceedings of PAC'03. Portland, (USA) 2003.

[10] W. Fischer and U. Iriso. "Bunch patterns and pressure rise in RHIC". BNL C-A/AP/118 (2003).

[11] M. Blaskiewicz et al. "Electron cloud instabilities in the PSR and SNS". PRST-AB 6, 014203. Jan. 2003. 
[12] Y. Cai, M. Furman et M. Pivi. "Build up of Electron Cloud with Different Bunch Pattern in the Presence of Solenoid Field". Proceedings of PAC'03. Portland, (USA) 2003.

[13] M. Furman and G.R. Lambertson. "The Electron Cloud instability in PEPII: an update". Proceedings of PAC'97, Vancouver (Canada), 1997.

[14] R.J. Macek. "Possible cures to the electron cloud problems". Proceedings of ECLOUD'02. Geneva, (Switzerland), April 2002.

[15] J. Gullotta, D. Gassner, et al. "RHIC Electron Detector Signal Processing Design". Proceedings of PAC'03. Portland, (USA) 2003.

[16] S.Y. Zhang, H. C. Hseuh and T. Roser. "NEG Coating at RHIC". CA/AP/99 (2003). 